\title{
Adaptive Power Transformer Lifetime Predictions through Machine Learning \& Uncertainty Modelling in Nuclear Power Plants
}

\author{
Jose Ignacio Aizpurua, Member, IEEE, Stephen D. J. McArthur, Fellow, IEEE, Brian G. \\ Stewart, Member, IEEE, Brandon Lambert, James G. Cross, and Victoria M. Catterson Senior Member, IEEE,
}

\begin{abstract}
The remaining useful life (RUL) of transformer insulation paper is largely determined by the winding hotspot temperature (HST). Frequently the HST is not directly monitored and it is inferred from other measurements. However, measurement errors affect prediction models and if uncertain variables are not taken into account this can lead to incorrect maintenance decisions. Additionally, existing analytic models for HST calculation are not always accurate because they cannot generalize the properties of transformers operating in different contexts. In this context, this paper presents a novel transformer condition assessment approach integrating uncertainty modeling, datadriven forecasting models and model-based experimental models to increase the prediction accuracy and handle uncertainty. The proposed approach quantifies the effect of measurement errors on transformer RUL predictions and confirms that temperature and load measurement errors affect the RUL estimation. Forecasting results show that the extreme gradient boosting (XGB) algorithm best captures the non-linearities of the thermal model and improves the prediction accuracy amongst a number of forecasting approaches. Accordingly, the XGB model is integrated with experimental models in a Particle Filtering framework to improve thermal modelling and RUL prediction tasks. Models are tested and validated using a real dataset from a power transformer operating in a nuclear power plant.
\end{abstract}

Index Terms-Condition assessment, forecasting, prognostics and health management, sensitivity, transformers.

\section{INTRODUCTION}

$\mathbf{P}$ OWER transformers are critical assets in the power grid. Condition monitoring and maintenance planning of transformers is crucial because their failure can lead to lack of export capability or even to catastrophic failures [1], [2]. With the increase of monitored parameters prognostics and health management (PHM) strategies have emerged as effective solutions to identify early indicators of anomalies, diagnose faults and predict the remaining useful life (RUL) of power assets [3]. The operation context of different transformers determines the best PHM strategy for monitoring their health [4]. This paper focuses on nuclear power plants (NPPs) and the aging of transformers in this context is affected by NPP operation.

J. I. Aizpurua, S. D. J. McArthur and B. G. Stewart are with the Institute of Energy \& Environment, University of Strathclyde, Glasgow, UK (e-mail: jose.aizpurua@strath.ac.uk; s.mcarthur@strath.ac.uk; brian.stewart.100@strath.ac.uk).

B. Lambert is with Bruce Power, Kincardine, Canada (e-mail: brandon.lambert@brucepower.com).

James G. Cross is with Kinectrics Inc., Toronto, Canada (e-mail: james.cross@kinectrics.com).
Transformer loading capability and RUL depend on the thermal conditions, and at the same time, thermal conditions depend on the load, environmental conditions and transformer parameters [5]. The winding hot-spot temperature (HST) is the main factor that determines the RUL of the insulation paper. The paper is comprised of polymers, in which the number of monomers, also known as degree of polymerization (DP), determines the strength and RUL of the paper [6]. A DP value of 200 is considered the end of life (EOL) condition of the solid paper [7]. The calculation of the HST is complex and it can be affected by other accelerating factors such as moisture, furans, carbon dioxide, or carbon monoxide [8]. For instance, the presence of moisture under high loading conditions can lead to bubble formation and potential catastrophic failure [8], [9]. Underestimated HST may lead to reduced cooling system operation and the transformer could be running hotter with an accelerating aging rate and significant reduced lifetime.

The IEEE C57.91 standard presents an analytic model to calculate the HST [7]. However, this model may not be generally applicable for all transformers operating in different contexts [10]-[12], and accordingly there have been proposed different machine learning methods which create fit-forpurpose thermal prediction models such as Artificial Neural Networks (ANN) [13], the C57.91 model with error correction via ANN [14], fuzzy logic with ANN [15], an evolving Gaussian fuzzy system [16], genetic programming [10], and ensemble of quantile regression models [17]. Temperature predictions can refer to different time horizons including short (few minutes), medium (few hours) or long term (few days) and the prediction error increases with the prediction horizon [14], [17]. The models mentioned above focus on short-tomedium term predictions. This paper focuses on mediumto-long term predictions because the proposed framework is planned to operate in an NPP and the decision window needs to be long enough to adopt timely decisions.

Additionally, none of these models go beyond thermal modelling to quantify the transformer RUL. Among those that model transformer RUL, some authors have used statistical distributions, e.g. Perk's model [18], Weibull distribution [19] or lognormal distribution [20], while others have used experimental models such as the Arrhenius equation in C57.91 for transformer utilisation improvement through dynamic rating [21] or RUL predictions [22]. The focus of this paper is on the second group due to the interest in insulation paper. Among those that consider experimental models and inspection data, 
there is no consideration of improved thermal models, and default thermal equations in C57.91 are used for transformer RUL calculation.

The estimation of transformer aging parameters is complex and non-deterministic because the heat transfer process is distributed over different surfaces in the winding and insulation structures and there may be measurement errors. Accordingly, uncertainty modelling is critical for well-informed predictions. For instance, Jauregui-Rivera et al. [23] used bootstrapping methods to quantify confidence intervals for thermal parameters. Some of the reviewed models consider uncertainties corresponding to different measured values [8], [17], [20], [22] and there are others which integrate different transformer health assessment parameters through an uncertainty-aware evidential reasoning framework [24]. However, to the best of our knowledge, there is no approach which integrates datadriven thermal forecasting models with model-based lifetime experimental models to increase prediction accuracy and handle uncertainty. This would help engineers in decision-making with error measurements and predicting the effect of future scenarios with varying conditions on RUL with more accurate results than experimental models.

Accordingly, this work presents a Bayesian inference framework to quantify the uncertainty-informed RUL and analyse the effect of measurement errors on RUL estimation. Building on this framework, an improved transformer RUL prediction approach is proposed integrating machine learning and experimental models in the Bayesian framework. Therefore the contributions of this paper are (i) the evaluation of the sensitivity of the effect of measurement errors on transformer RUL estimation, and (ii) adaptive prognostics predictions through the integration of uncertainty modelling, forecasting models and IEEE lifetime models.

The rest of the paper is organized as follows. Section II presents the IEEE thermal and lifetime models and analyses the uncertainty sources. Section III presents the proposed approach for uncertainty-aware predictive modelling. Section IV presents case study results and finally, Section V concludes.

\section{TRANSFORMER THERMAL \& LIFETIME MODELLING}

The IEEE C57.91 standard defines the insulation paper aging acceleration factor at time $t, F_{\mathrm{AA}}(t)$, as [7]:

$$
F_{\mathrm{AA}}(t)=e^{\frac{15000}{383}-\frac{15000}{273+\Theta_{\mathrm{H}}(t)}}
$$

where $\Theta_{\mathrm{H}}(t)$ is the transformer winding's HST at time $t$ in ${ }^{\circ} \mathrm{C}$, which can be calculated from other measurements [7]:

$$
\begin{aligned}
\Theta_{\mathrm{H}}(t) & =\Theta_{\mathrm{TO}}(t)+\Delta \Theta_{\mathrm{TO}, \mathrm{H}}(t) \\
& =\Theta_{\mathrm{A}}(t)+\Delta \Theta_{\mathrm{A}, \mathrm{TO}}(t)+\Delta \Theta_{\mathrm{TO}, \mathrm{H}}(t)
\end{aligned}
$$

where $\Theta_{\mathrm{A}}(t)$ and $\Theta_{\mathrm{TO}}(t)$ are the ambient temperature and topoil temperature (TOT) at time instant $t$ and $\Delta \Theta_{\mathrm{A}, \mathrm{TO}}(t)$ and $\Delta_{\mathrm{TO}, \mathrm{H}}(t)$ are the TOT and HST rise over ambient temperature and TOT respectively at time $t$ calculated through:

$$
\begin{aligned}
& \Delta \Theta_{\mathrm{A}, \mathrm{TO}}(t)=\left(\Delta \Theta_{{\mathrm{A}, \mathrm{TO}_{\mathrm{u}}}}(t)-\Delta \Theta_{\mathrm{A}, \mathrm{TO}_{\mathrm{i}}}(t)\right)\left(1-e^{-\frac{\Delta t}{\tau} \mathrm{TO}}\right)+\Delta \Theta_{{\mathrm{A}, \mathrm{TO}_{\mathrm{i}}}(t)} \\
& \Delta \Theta_{\mathrm{TO}, \mathrm{H}}(t)=\left(\Delta \Theta_{\mathrm{TO}, \mathrm{H}_{\mathrm{u}}}(t)-\Delta \Theta_{\mathrm{TO}, \mathrm{H}_{\mathrm{i}}}(t)\right)\left(1-e^{-\frac{\Delta t}{\tau} \mathrm{H}}\right)+\Delta \Theta_{\mathrm{TO}, \mathrm{H}_{\mathrm{i}}}(t)
\end{aligned}
$$

where $\tau_{\mathrm{TO}}$ and $\tau_{\mathrm{H}}$ are oil and winding time constants, $\Delta t$ is the loading time interval, $\Delta \Theta_{\mathrm{A}, \mathrm{TO}_{\mathrm{i}}}(t)$ and $\Delta \Theta_{\mathrm{TO}, \mathrm{H}_{\mathrm{i}}}(t)$ are the initial TOT and HST rise over ambient and TOT respectively at time $t$, and $\Delta \Theta_{\mathrm{A}_{,} \mathrm{TO}_{\mathrm{u}}}(t)$ and $\Delta \Theta_{\mathrm{TO}, \mathrm{H}_{\mathrm{u}}}(t)$ are the ultimate TOT and HST rise over ambient and TOT respectively at time $t$, defined as:

$$
\begin{aligned}
& \Delta \Theta_{\mathrm{A}, \mathrm{TO}_{\mathrm{u}}}(t)=\Delta \Theta_{\mathrm{TO}, \mathrm{R}} \cdot\left[\left(\left(i(t) / i_{\mathrm{r}}\right)^{2} \gamma+1\right) /(\gamma+1)\right]^{n} \\
& \Delta \Theta_{\mathrm{TO}, \mathrm{H}_{\mathrm{u}}}(t)=\Delta \Theta_{\mathrm{H}, \mathrm{R}} \cdot\left(i(t) / i_{\mathrm{r}}\right)^{2 m}
\end{aligned}
$$

where $\gamma$ is the ratio of load loss at rated load to loss at zero load, $i(t)$ is the transformer load at time $t, i_{\mathrm{r}}$ is the rated load, $\Delta \Theta_{\mathrm{TO}, \mathrm{R}}$ and $\Delta \Theta_{\mathrm{H}, \mathrm{R}}$ are the TOT and HST rise at rated load respectively, and $m$ and $n$ are transformer parameters determined through a lookup table depending on the cooling system of the transformer [7].

In order to determine the RUL at time $t, R U L(t)$, Eq. (1) can be converted into a Markovian recurrence relation form, where the insulation paper health state depends only on its previous state and current conditions:

$$
R U L(t)=R U L(t-1)-F_{\mathrm{AA}}(t)=R U L(t-1)-e^{\left(\frac{15000}{383}-\frac{15000}{273+\Theta_{\mathrm{H}}^{(t)}}\right)}
$$

At instant $t=0, R U L(t-1)$ equals to the initial lifetime estimation $R U L_{0}$. In subsequent iterations $R U L_{0}$ is updated with the most up-to-date RUL estimation to reflect the previous state at $t-1$. Eq. (5) relates the insulation paper RUL with temperature and load measurements and it guides the loading capability of the transformer by examining the effect of different load profiles on the transformer RUL. However, the application of (5) gives a single RUL value at time $t$ and it does not consider the effect of different uncertainties such as measurement errors that affect the RUL estimation.

\section{A. Sources of uncertainty}

The HST is inferred from indirect measurements [cf. Eq. (2)]. Assuming that the TOT is measured, then HST calculated from TOT measurements may include measurement errors of TOT and load sensors. Additionally, the initial health state and the paper consumption process [cf. Eq. (5)] may not be accurate due to lack of knowledge and other factors involved in the paper degradation process.

If these uncertainty-surrounded values are not considered, the HST estimation may lead to erroneous results. Therefore, the effect of measurement errors requires to be explicitly considered. Accordingly, (2) including measurement errors and assuming steady-state $[\Delta t=0$ in (3)] is converted into:

$$
\Theta_{\mathrm{H}}(t)=\left(\Theta_{\mathrm{TO}}(t)+\varphi_{\mathrm{TO}}\right)+\Delta \Theta_{\mathrm{H}, \mathrm{R}} \cdot\left[\left(i(t)+\varphi_{\mathrm{i}}\right) / i_{\mathrm{r}}\right]^{2 m}
$$

where $\varphi_{\mathrm{TO}}$ denotes the top-oil measurement error and $\varphi_{\mathrm{i}}$ designates the load measurement error.

Similarly, the paper degradation process in (5) is not a deterministic process, and it also needs to integrate uncertainty information corresponding to this process [22]:

$$
R U L(t)=R U L(t-1)+w_{\mathrm{RUL}_{\mathrm{t}-1}}-e^{\left(15000+w_{\mathrm{t}}\right)\left(\frac{1}{383}-\frac{1}{273+\Theta_{\mathrm{H}}(t)}\right)}
$$

where $w_{\mathrm{RUL}_{\mathrm{t}-1}}$ denotes the uncertainty of the lifetime estimation at $t-1, w_{\mathrm{t}}$ denotes the degradation process uncertainty and $\Theta_{\mathrm{H}}(t)$ is defined in (6). Initially $w_{\mathrm{RUL}}$ will denote the initial lifetime estimation error, $w_{\mathrm{RUL}_{0}}$, which will be propagated 
in subsequent iterations through the recurrence relation form of (7). Comparing (5) with (7) it is possible to see that the different uncertainty sources may affect the HST and RUL predictions. The proposed framework below effectively integrates these sources of uncertainty.

\section{Proposed Approach for Analytics-updated \& UNCERTAINTY-AWARE LIFETIME ANALYSIS}

The goal of the proposed framework is to estimate the current transformer insulation health state given inspection data up to now (diagnostics) and predict the likely future remaining lifetime given hypothetical future profiles (prognostics). Fig. 1 shows the PHM analysis framework where $\left\{z_{1}, \ldots, z_{\mathrm{i}}, \ldots, z_{\mathrm{k}}\right\}$ is the inspection data up to the current time instant $t_{\mathrm{k}}$ and $\mathrm{EOL}_{\mathrm{i}}$ denotes end of life due to to the i-th degradation trajectory.

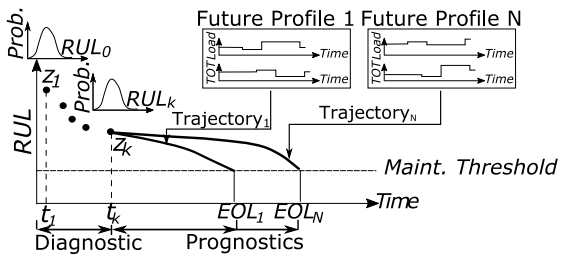

Fig. 1. Transformer insulation paper PHM framework

The generalization and accuracy of the HST model in (2) can be enhanced by complementing the equation with forecasting models. The analytic equations used to quantify $\Theta_{\mathrm{TO}}(t)$ are not always accurate [10], [14] because it is difficult to generalize with an analytic relation the properties of different transformers operating in different contexts and this affects transformer lifetime estimation. Accordingly, a novel RUL prediction framework is proposed shown in Fig. 2.

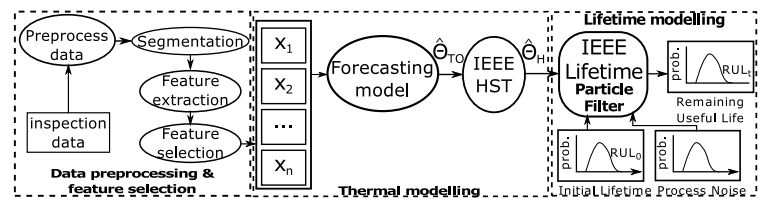

Fig. 2. Proposed remaining paper lifetime framework

The inspection data is not directly processable because it may include outliers and noisy measurements. The data preprocessing step denoises and filters the data. Subsequently the segmentation step divides the time series into different equidistant time periods. The preprocessed and segmented data is then connected with a feature extraction step so that a number of time-domain features are extracted. Finally, this stage is completed by selecting the most representative features for subsequent thermal and lifetime modelling steps (see Subsection III-A).

The thermal modelling approach is comprised of top-oil and HST calculations. For a number of utilities it is common not to have HST measurements because the required sensors are not cost-effective. Accordingly, machine learning (ML) techniques have been used to learn a predictive model which is able to predict the top-oil temperature, $\hat{\Theta}_{\mathrm{TO}}$, given a number of input parameters (see also Subsection III-B). Then this model is used along with the IEEE experimental model to estimate the HST:

$$
\hat{\Theta}_{\mathrm{H}}(t)=\hat{\Theta}_{\mathrm{TO}}(t)+\Delta \Theta_{\mathrm{TO}, \mathrm{H}}(t)
$$

The only difference between (8) and (2) is that the TOT is predicted using ML models and not using analytic equations.

After implementing the HST prediction model, it is possible to embed it into the lifetime modelling framework through the $\mathrm{PF}$ approach for a more accurate lifetime estimation which includes different uncertainty criteria (see Subsection III-C).

\section{A. Data pre-processing \& feature selection}

After denoising and filtering the measurements performed on-site (see Section IV), Fig. 3 shows the correlation of the top-oil temperature (vertical axis) with cooling water temperature, load and ambient temperature (horizontal axis), where the grey points are the actual data samples. The higher the density (plotted in red) the more likely is the correlation and vice-versa (plotted in green). For instance, the most likely condition for water temperature versus top-oil temperature (Fig. 3 left) is that for water temperature between $0^{\circ} \mathrm{C}$ and $5^{\circ} \mathrm{C}$ the top-oil temperature is concentrated at $25^{\circ} \mathrm{C}$ with a probability density value of 0.015 . As the water temperature increases up to $10^{\circ} \mathrm{C}$, the top-oil temperature fluctuates between $20^{\circ} \mathrm{C}$ and $35^{\circ} \mathrm{C}$ with a probability density value of 0.001 (green area in Fig. 3 left).

It can be seen in Fig. 3 that there is a non-linear relationship among the measured variables and the top-oil temperature. This indicates that these variables may be good predictors for top-oil temperature because they add new information to the forecasting model. This confirms expert knowledge [7] which states that for water-cooled transformers, the relevant parameters to estimate top-oil temperature are cooling water temperature, ambient temperature and load.

In order to improve the prediction capability of forecasting models, it is possible to infer additional time-domain features from water temperature, ambient temperature and load timeseries. To this end, first the time series is divided into segments, and then features are inferred. Different state-of-the-art features have been extracted (listed in Table I [25], [26]) which results in a total of 18 features plus the three time series.

TABLE I

TIME-DOMAIN FEATURES OF THE $k$-TH SEGMENT OF THE INPUT DATA CONTAINING A TOTAL OF $N$ DATA SAMPLES, $x_{i, k}$, PER SEGMENT $k$

\begin{tabular}{|lclc|}
\hline Feature & \multicolumn{1}{c}{ Definition } & Feature & Definition \\
\hline Mean & $X_{1}=\frac{\sum_{i=1}^{N} x_{\mathrm{i}, \mathrm{k}}}{N}$ & $\begin{array}{l}\text { Impulse } \\
\text { factor }\end{array}$ & $X_{2}=\frac{\max \left(\left|x_{\mathrm{i}, \mathrm{k}}\right|\right)}{\frac{1}{N} \Sigma_{i=1}^{N}\left|x_{\mathrm{i}, \mathrm{k}}\right|}$ \\
\hline Skewness & $X_{3}=\frac{\Sigma_{i=1}^{N}\left(x_{\mathrm{i}, \mathrm{k}}-\mu_{\mathrm{k}}\right)^{3}}{(N-1) \sigma_{\mathrm{k}}{ }^{3}}$ & Kurtosis & $X_{4}=\frac{\Sigma_{i=1}^{N}\left(x_{\mathrm{i}, \mathrm{k}}-\mu_{\mathrm{k}}\right)^{4}}{(N-1) \sigma_{\mathrm{k}}}$ \\
\hline $\begin{array}{l}\text { Root } \\
\text { mean } \\
\text { square }\end{array}$ & $X_{5}=\sqrt{\frac{\sum_{i=1}^{N} x_{i, k}^{2}}{N}}$ & $\begin{array}{l}\text { Crest } \\
\text { factor }\end{array}$ & $X_{6}=\frac{\max \left(\left|x_{\mathrm{i}, \mathrm{k}}\right|\right)}{\sqrt{\frac{1}{N} \Sigma_{i=1}^{N} x_{i, k}^{2}}}$ \\
\hline
\end{tabular}

The thermal properties of the oil suggest that there may be a delay in the heat transfer process and accordingly lagged signals may be useful to improve the accuracy of predictions. However, in this case, the use of lagged signals for each of these temperatures does not improve the accuracy of top-oil temperature predictions. The prediction tasks of this work are focused on a long term horizon with highly non-linear signals (see Subsection IV-B2). Then the use of e.g. a top-oil lagged signal involves feeding back the top-oil predictions. This recursive mechanism has a negative effect over a longterm prediction window by accumulating and propagating the prediction errors and worsening the final prediction. In any case, one of the implemented ML models explicitly takes into 

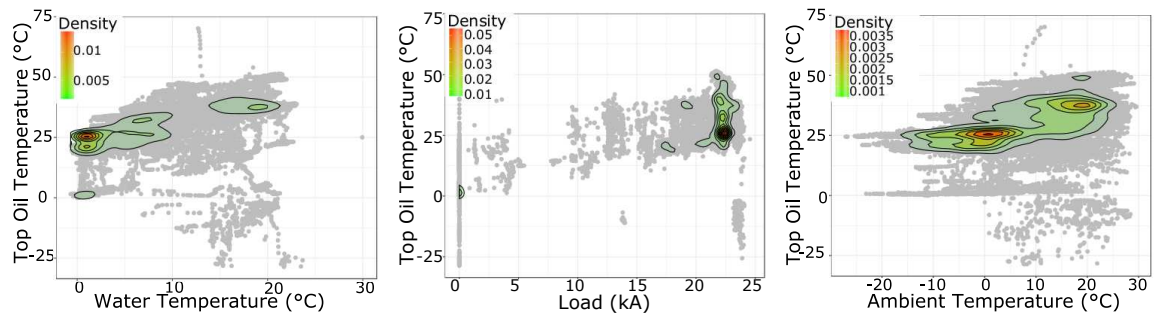

Fig. 3. Top-oil temperature correlated with cooling water temperature, load and ambient temperature

account lagged signals and their effect on the prediction (see LSTM models in Subsection III-B4).

The feature selection process is implemented in Section IV-B1 using the case study datasets. Accordingly, the design of the subsequently introduced machine learning algorithms are based on these datasets and extracted features.

\section{B. Thermal modelling through machine learning methods}

So as to forecast the top-oil temperature, different $\mathrm{ML}$ models have been designed and tested including Random Forests (RF) [27], Artificial Neural Networks (ANN) [28] and Support Vector Regression (SVR) [29], and also improved versions of RF (Extreme Gradient Boosted Regression Tree, XGB [30]) and ANN (Long Short Term Memory, LSTM [31]). The rationale for choosing these models is to compare the predictive power of XGB and LSTM with their counterpart models (RF, ANN) and other classical models (SVR).

1) Random Forests $(R F): \mathrm{RF}$ is an ensemble of recursive trees [27]. Each tree is generated from a bootstrapped sample and a random subset of descriptors is used at the branching of each node in the tree. RF creates a large number of trees by repeatedly resampling training data and averaging differences through voting.

The RF model has been implemented through the randomForest package in $\mathrm{R}$. The hyperparameters include the number of trees (ntree) and the number of variables randomly sampled as candidates at each split (mtry). These parameters have been optimized through a 10 repeated 5 fold cross-validation $(\mathrm{CV})$ process searching the best parameters from a predefined grid of parameters (see Subsection IV-B2 for more details of the CV process): ntree $=[100,500,1000,1500]$ and $m$ try $=[1: 15]$. Best results were obtained with ntree $=500$ and mtry $=2$.

2) Extreme Gradient Boosted Regression Tree (XGB): XGB [30] is a faster and more efficient implementation of gradient boosting [32] which creates an accurate learner by combining many regression trees. The objective of training an XGB model is to minimize the training loss and avoid overfitting through regularization terms. This process is based on additive training implemented through a second order gradient algorithm [30].

The XGB model has been implemented through the xgbtree package in $\mathrm{R}$. The hyperparameters include the maximum depth of the tree (max_depth) and learning rate $(\eta)$. The more complex the tree, the more complicated patterns it will learn, but it will be more prone to over-fitting. The learning rate models the error generalization. These hyperparameters have been optimized through a 10 repeated 5- fold CV process searching the optimal parameters from a predefined grid of parameters: $\eta=[0.001,0.003,0.01,0.1,0.3]$, max_depth $=[1,2,4,6,10]$. The best parameters for this work are $\eta=0.3$ and max_depth $=2$.

3) Artificial Neural Network (ANN): ANNs are widely used for classification and regression tasks [28]. The multilayer perceptron (MLP) feedforward model was used in this work. The MLP is a three-layer network (input, hidden, output) comprised of fully connected neurons. Each neuron performs a weighted sum of its inputs and passes the results through an activation function. All the designed ANN models use a sigmoid activation function for the hidden layer and linear activation function for output nodes.

Model training is performed using a back-propagation algorithm. The goal is to learn the neuron weights to generate the network output from the sample input, which minimizes the error with respect to the target output. 10 repeated 5 fold CV was used to select an optimal number of hidden nodes. A number of networks were trained for each fold varying the number of hidden nodes from 1 up to 30. Of the trained networks, the one with the highest accuracy was selected and was comprised of 13 hidden nodes. The ANN was implemented using the nnet $\mathrm{R}$ package.

4) Long Short Term Memory (LSTM): Is a type of recurrent neural network which can capture correlations among signals which involve long or short term time lags [31]. An LSTM model is comprised of one input layer, one or more recurrent hidden layers, and one output layer. The recurrence loop allows layers to store information. Instead of using nodes for the hidden layer as in ANN models, the basic units of LSTM models are cells, which can perform complex logic operations (sometimes resembling finite state machines). The LSTM model is trained through back-propagation of errors using stochastic gradient descent.

The LSTM model has been implemented through the Keras package in Python. The hyperparameter tuning process consists of selecting the next parameters: number of layers, number of LSTM units, batch size, learning rate, and number of epochs. Batch size denotes the subset size of the training data. The LSTM model is not trained in a single trial, but takes subsets of the data and learning correlations between subsets. Each batch trains a network in successive order taking into account the updated weights coming from the previous batch. Number of epochs is the number of forward and backward passes of all the training data.

The number of hidden layers was fixed to a maximum of three layers and a number of different configurations with 
different hyperparameters were tested through 10 repeated 5-fold CV and grid search with parameters defined in the following ranges: batch size $=[5,10,15,30,45,90]$, number of cells $=[1: 30]$, epochs $=[10,1000,2000,4000,5000,10000]$, activation functions $=$ [softmax, relu, linear, tanh, sigmoid, softsign, softplus $]$, learning rate $=[1 \mathrm{e}-4,1.5 \mathrm{e}-4,2 \mathrm{e}-4,3 \mathrm{e}-4$, $4 \mathrm{e}-4,5 \mathrm{e}-4,1 \mathrm{e}-3,2 \mathrm{e}-3]$. Best results were obtained with two layers with 7 cells in each layer, batch size of 15, 5000 epochs, learning rate of $1.5 \mathrm{e}-4$ and softmax activation function.

5) Support Vector Regression (SVR): The SVR maps input data into an $m$-dimensional feature space using a kernel function [29]. The kernel translates a nonlinearly separable problem into a feature space, which is linearly separable by a hyperplane. The SVR defines a $\epsilon$ loss function that ignores the errors situated within a certain distance of the true value.

The SVR is parametrized through the choice of kernel function. For a nonlinear problem the RBF kernel is recommended: $k\left(x, x^{\prime}\right)=\exp \left(\gamma\left\|x-x^{\prime}\right\|^{2}\right)$, where $\gamma$ is the $\mathrm{RBF}$ width, $x$ and $x^{\prime}$ are training and testing data samples, and $\|d\|$ is the Euclidean norm. The SVR solves an optimization problem maximizing the distance from the hyperplane to the nearest training point. SVR penalizes the loss function with a cost variable $c$. SVR training consists of calculating the hyperparameters $c$ and $\gamma$. Model training was performed using the $\mathrm{R}$ kernlab and MLR packages and grid search was used to optimize $c$ and $\gamma$ within $\mathrm{c}=\left[2^{-8}, 2^{-4}, 2^{-2}, 1\right]$ and $\gamma=\left[2^{-8}: 2: 2^{4}\right]$. Of the trained SVRs, the one with the highest accuracy was selected which had $\epsilon=0.1, \gamma=0.25$ and $c=1$

\section{Lifetime modelling through Particle Filtering (PF)}

$\mathrm{PF}$ is a Monte Carlo based Bayesian filtering method. PF enables the integration of multiple measurements in a single degradation model $f(\cdot)$ and filters the true state of the system, $x_{t}$, taking into account multiple sources of uncertainty [33]. A two-step method is implemented when PF is used for PHM [34]. Firstly the system state estimation is performed:

$$
\begin{gathered}
x_{\mathrm{k}}=f\left(x_{\mathrm{k}-1}, w_{\mathrm{k}-1}\right) \\
z_{\mathrm{k}}=h\left(x_{\mathrm{k}}, \varphi_{\mathrm{k}}\right)
\end{gathered}
$$

where $f(\cdot)$ is the state degradation function, $w_{\mathrm{k}}$ is a state noise vector $w_{\mathrm{k}}=\left\langle w_{\mathrm{t}}, w_{\mathrm{RUL}_{\mathrm{t}}}\right\rangle, h(\cdot)$ is the measurement function, and $\varphi_{\mathrm{k}}$ is a measurement noise vector $\varphi_{\mathrm{k}}=\left\langle\varphi_{\mathrm{TO}}, \varphi_{\mathrm{i}}\right\rangle$.

Fig. 4 shows the application of (9) and (10) in the transformer paper RUL estimation process. The measurement function defined in (6) integrates load $(i)$ and top-oil temperature $\left(\Theta_{\mathrm{TO}}\right)$ along with their measurement errors $\left(\varphi_{\mathrm{i}}\right.$ and $\left.\varphi_{\mathrm{TO}}\right)$ and other transformer parameters, and computes the hotspot temperature $\Theta_{\mathrm{H}}$. The degradation function defined in (7) integrates the process noise $W_{\mathrm{t}}$ and calculates the RUL from the HST and initial health state. The initial health state is then iteratively updated with the actual state.

The state estimation $x_{\mathrm{k}}$ given measurements $z_{\mathrm{k}}$ up to the time instant $k$ is defined in terms of probability density function (PDF) $p\left(x_{\mathrm{k}} \mid z_{0: \mathrm{k}}\right)$. The initial state $p\left(x_{0}\right)$ is assumed to be known (see diagnostics in Fig. 1). There are different methods to estimate the transformer's initial health state such as the experimental analysis of the degree of polymerization of the insulation paper, or if the paper is new, the initial

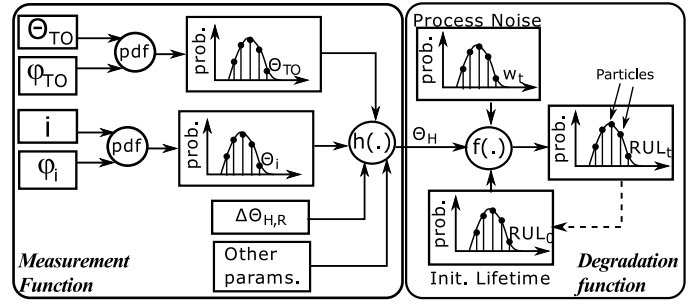

Fig. 4. PF framework for transformer insulation paper analysis

health state may be assumed to be of 180000 hours under the conditions stated in IEEE C57.91 [7]. The prior PDF of the state $x_{\mathrm{k}}$ from the distribution $p\left(x_{\mathrm{k}-1} \mid z_{0: \mathrm{k}-1}\right)$ is determined by:

$$
\begin{aligned}
p\left(x_{\mathrm{k}} \mid z_{0: \mathrm{k}-1}\right) & =\int p\left(x_{\mathrm{k}} \mid x_{\mathrm{k}-1}, z_{0: \mathrm{k}-1}\right) p\left(x_{\mathrm{k}-1} \mid z_{0: \mathrm{k}-1}\right) d x_{\mathrm{k}-1} \\
& =\int p\left(x_{\mathrm{k}} \mid x_{\mathrm{k}-1}\right) p\left(x_{\mathrm{k}-1} \mid z_{0: \mathrm{k}-1}\right) d x_{\mathrm{k}-1}
\end{aligned}
$$

where the state-transition distribution function $p\left(x_{\mathrm{k}} \mid x_{\mathrm{k}-1}\right)$ is defined by the recurrence relation form in (9). In order to update the prior $P D F$, a new measurement is collected at time $k ; z_{\mathrm{k}}$, and the posterior PDF is obtained using the Bayes rule:

$$
p\left(x_{\mathrm{k}} \mid z_{0: \mathrm{k}}\right)=\frac{p\left(x_{\mathrm{k}} \mid z_{0: \mathrm{k}-1}\right) p\left(z_{\mathrm{k}} \mid x_{\mathrm{k}}\right)}{p\left(z_{\mathrm{k}} \mid z_{0: \mathrm{k}-1}\right)}
$$

The analytic solution of (12) is complex. Thus, the PF was proposed based on iterative application of prediction, update and resampling steps at each time instant $k$ [33].

Prediction: assuming at time $k-1, N_{\mathrm{p}}$ random samples (particles) of the system state are available, $\left\{x_{k-1}^{i}\right\}_{i=1}^{N_{\mathrm{p}}}$, as a realization of the posterior distribution $p\left(x_{\mathrm{k}-1} \mid z_{0: \mathrm{k}-1}\right)$, the prediction at $k$ is performed by sampling the probability distribution of the system noise $w_{\mathrm{k}-1}$ and simulating the system dynamics according to (9) to generate new samples $x_{k}^{i}$ which are realizations of the predicted distribution $p\left(x_{\mathrm{k}} \mid z_{0: \mathrm{k}-1}\right)$.

Update: each sampled particle is assigned a weight based on the likelihoods of observations $z_{\mathrm{k}}$ collected at time $k$ :

$$
w_{k}^{i}=\frac{p\left(z_{\mathrm{k}} \mid x_{k}^{i}\right)}{\sum_{j=1}^{N_{\mathrm{p}}} p\left(z_{\mathrm{k}} \mid x_{k}^{j}\right)}
$$

An approximation of the posterior PDF $p\left(x_{\mathrm{k}} \mid z_{0: \mathrm{k}}\right)$ is then obtained from the weighted samples $\left\{x_{k}^{i}, w_{k}^{i}\right\}_{i=1}^{N_{p}}$.

Resampling: as the PF evolves in time, weight degeneracy phenomena occurs where all but one particle have negligible weights [33]. To avoid this problem a degeneracy condition is defined based on the effective size:

$$
\hat{N}_{\text {eff }}=1 / \sum_{i=1}^{N_{\mathrm{p}}} w_{k}^{i}
$$

If $\hat{N}_{\text {eff }}$ falls below a threshold $N_{\mathrm{T}}\left(N_{\mathrm{T}}=N_{\mathrm{p}} / 2\right.$ in this work), a systematic resampling step is applied [33].

Algorithm 1 below is a variant of the PF framework and defines the model implemented in this work for transformer paper lifetime modelling as defined in Fig. 2.

\section{CAse Study}

The health of transformers is critical for the NPP. The parameters of the main output transformer analyzed in this section are reported in Table II. It is assumed that a Normal 


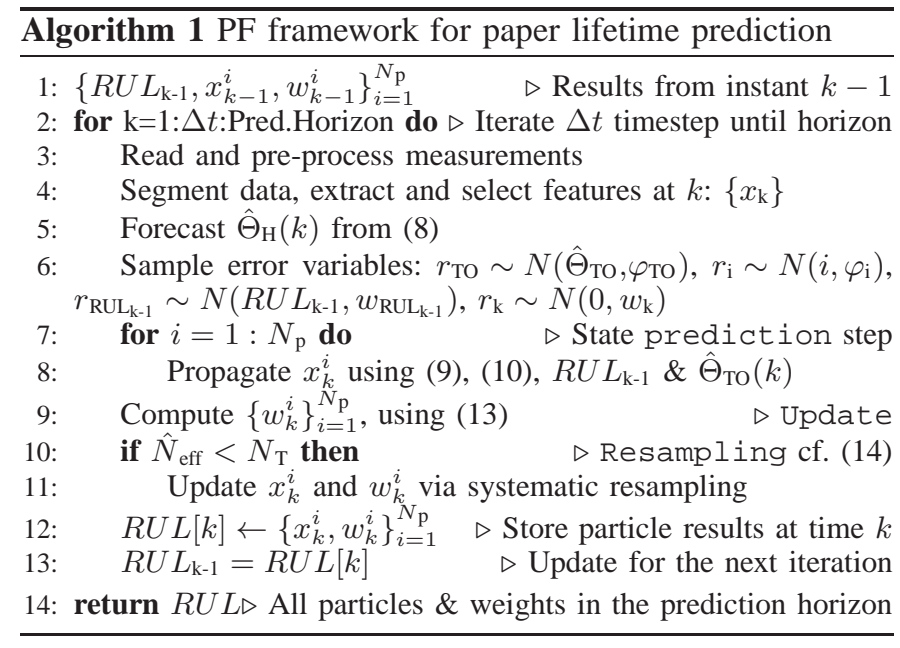

distribution models the uncertainty-related variables (cf. Algorithm 1, line 6). For confidentiality reasons, it is assumed that the initial state of the insulation paper is equal to a new paper with 180000 hours of life and an uncertainty of 500 hours, i.e. $R U L_{0} \sim N(180000,500)$ [7] and the process noise is assumed to be $r_{\mathrm{k}} \sim N(0,20)$. However, note that the PF framework is flexible and it enables the integration of nonNormal distributions too.

TABLE II

TRANSFORMER PARAMETERS

\begin{tabular}{|c|c|c|c|}
\hline Param. & Value & Param. & Value \\
\hline Cooling $/ \mathrm{m}, \mathrm{n}$ & Oil Directed Water Forced $/ 1,1$ & Rating & $267 \mathrm{MVA}$ \\
\hline$\Delta_{\mathrm{H}, \mathrm{R}} / \Delta_{\mathrm{TO}, \mathrm{R}}$ & $30{ }^{\circ} \mathrm{C} / 24.3^{\circ} \mathrm{C}$ & $\mathrm{V}_{1} / \mathrm{V}_{2}$ & $17 \mathrm{kV} / 230 \sqrt{3} \mathrm{kV}$ \\
\hline$w_{\text {core,coil }} / w_{\text {tank }}$ & $95254 \mathrm{~kg} / 30617 \mathrm{~kg}$ & $i_{\mathrm{r}} / \gamma$ & $15.1 \mathrm{kA} / 0.25$ \\
\hline
\end{tabular}

\section{A. Diagnostics \& sensitivity analysis}

1) Diagnostics: Using the proposed framework in Fig. 2, it is possible to estimate the actual health state of the insulation paper by replacing line 5 of Algorithm 1 with real $\Theta_{\mathrm{TO}}(t)$ measurements and scoping the prediction horizon into the length of the available data.

Assuming that the initial health state corresponds to 11/2012 the health state after 3 years and 10 months is evaluated, i.e. at 09/2016. Accordingly, the available datasets for top-oil and load for the same period are processed so as to first calculate the HST [cf. (6)] and then estimate the RUL at 09/2016. Fig. 5 shows the preprocessed load and top-oil temperature data.
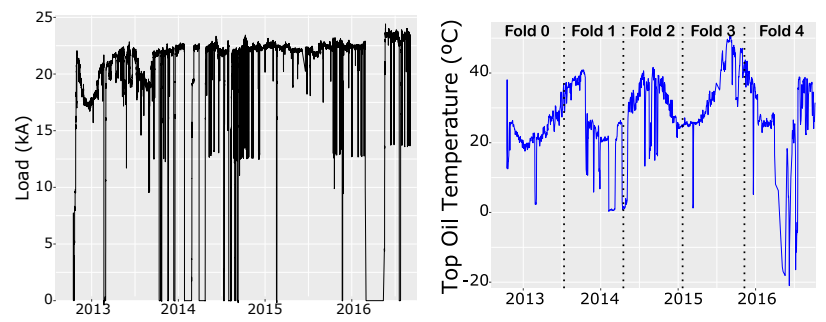

Fig. 5. Tested load and temperature profiles (11/2012 - 09/2016)

In order to calculate the health state of the transformer as of $09 / 2016$, it is assumed that the temperature error is $\varphi_{\mathrm{TO}}=$ $5{ }^{\circ} \mathrm{C}$ and the load error is $\varphi_{\mathrm{i}}=1 \mathrm{kA}$. The datasets in Fig. 5 are applied to the PF framework in Algorithm 1 as follows:

- Iine 1: initial state: $R U L_{0} \sim N(180000,500)$.
- Iine 5: calculate the HST from (2) using the collected top-oil temperature data in Fig. 5.

- line 6: draw random numbers corresponding to the selected error variables.

- Iines 7-13: calculate the PDF of the RUL at $t$.

This process is repeated for the selected prediction horizon (line 2) with a $\Delta t=1$ hour timestep, and finally the PDF of the RUL is obtained after processing all the available data (line 14). There is no need to extract features and predict the TOT because the TOT is available until 09/2016 and the goal is to diagnose the health state at this time instant.

Fig. 6 shows transformer lifetime diagnostics results. The operating time axis denotes the processed data (11/201209/2016), the RUL axis denotes the degradation of the health state starting from the initial state, and the density axis denotes the PDF value.

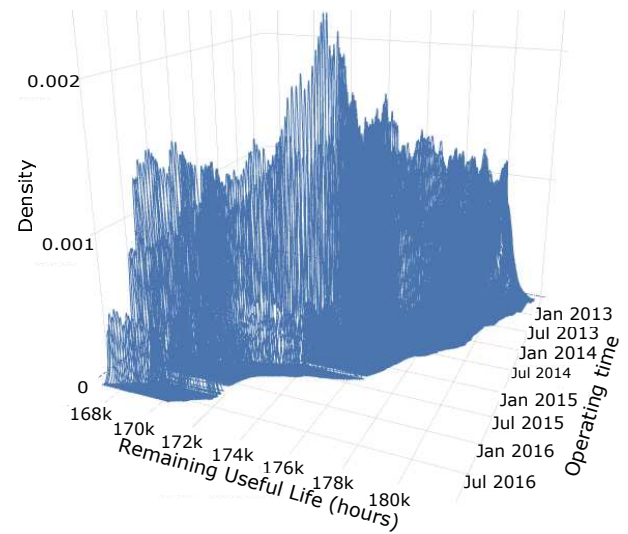

Fig. 6. Transformer diagnostics results for 11/2012-09/2016

Fig. 7 shows the health state of the transformer at the initial and final time instants directly taken from Fig. 6.

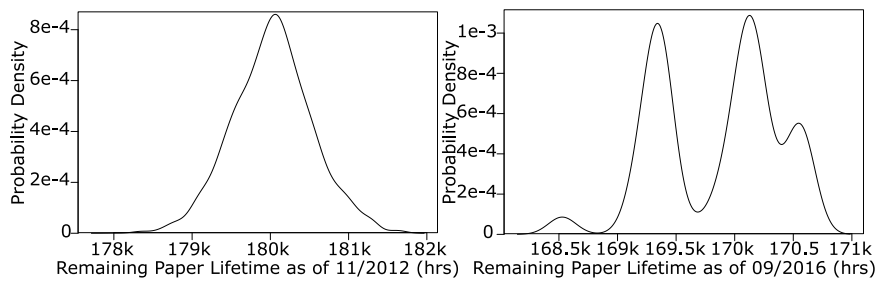

Fig. 7. RUL at initial and final time instants (inferred from Fig. 6)

An important result is that the initial assumptions about the Normal distribution of the errors (cf. Fig. 7 left) change as the $\mathrm{PF}$ framework propagates the measurements and associated errors. By the end of the diagnostics process (cf. Fig. 7 right) Normality cannot be assumed and therefore, when inferring the confidence intervals, standard percentile values are not applicable. Namely, the final health state is distributed into two nodes located at 169300 and 170200 hours. Accordingly, it is necessary to calculate the area under the curve so as to ensure that it covers the desired confidence interval (CI) area. Fig. 8 shows the $95 \%$ confidence interval quantification concept for non-Normal distributions bounded into [95- CI, 95 $\left.{ }^{+} \mathrm{CI}\right]$.

The inference of the confidence intervals facilitates the decision-making process because it enables engineers to adopt 


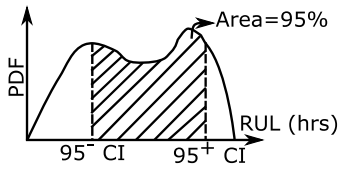

Fig. 8. $95 \%$ confidence intervals $\left[95^{-} \mathrm{Cl}, 95^{+} \mathrm{Cl}\right]$

an uncertainty-informed decision with intuitive lower and upper limits on the estimated parameters. Accordingly, Fig. 9 shows the maximum likelihood and 95\% CI of the predictions in Fig. 6.

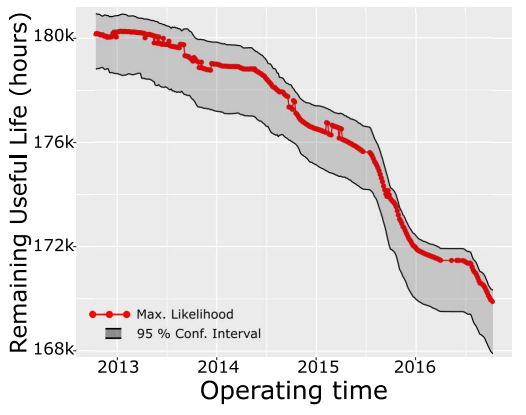

Fig. 9. Transformer diagnostics, 95\% Cl of Fig. 6

Fig. 9 shows the maximum likelihood and 95\% CI for the PDFs shown in Fig. 6. The degradation is almost exponential as determined by the ageing acceleration factor in (1), but this is affected by the de-energized periods of the transformer which are reflected in the load and top-oil temperature. For example, the transformer was shut down in mid-2016 which resulted in zero load, decreased top-oil temperature, and accordingly almost negligible RUL decrease. The uncertainty propagation is dependent on the assumed error variables and processed data as discussed in the next subsection.

2) Sensitivity Analysis: In order to evaluate the effect of error variables on the RUL estimation a sensitivity analysis has been performed examining the effect of the change of load and temperature measurement errors. Note that this information is lost with existing lifetime estimation models.

The HST in (6) defines the effect of load and temperature measurements errors. For this case study (cf. Table II), this equation is parametrized as follows $\left(i(t), \varphi_{\mathrm{i}}\right.$ are in $\mathrm{kA}$ units):

$$
\begin{aligned}
\Theta_{\mathrm{H}}(t) & =\Theta_{\mathrm{TO}}(t)+\varphi_{\mathrm{TO}}+\left[\Delta_{\mathrm{H}, \mathrm{R}} / i_{\mathrm{r}}^{2}\right] \cdot\left[i(t)^{2}+\varphi_{\mathrm{i}}^{2}+2 \cdot i(t) \cdot \varphi_{\mathrm{i}}\right] \\
& =\Theta_{\mathrm{TO}}(t)+\varphi_{\mathrm{TO}}+0.13 \cdot i(t)^{2}+0 \cdot 13 \cdot \varphi_{\mathrm{i}}{ }^{2}+0.13 \cdot i(t) \cdot \varphi_{\mathrm{i}}
\end{aligned}
$$

It is possible to see that the effect of temperature measurement errors are added as absolute values. In contrast, for small load variations, the effect of load measurement errors on the HST are not relevant. However, if $\varphi_{\mathrm{i}}>\sqrt{(1 / 0.13)} \sim 2.78$ then the effect starts increasing rapidly due to the factor $0.13 . \varphi_{\mathrm{i}}{ }^{2}$ and the exponential degradation in (7). The term 0.13.i(t). $\varphi_{\mathrm{i}}$ depends on the specific transformer loading.

The effects of different load and temperature errors have been analysed using monitored data. For computational efficiency the data has been limited to a year (11/2012-11/2013). Fig. 10 shows the effect of different load measurement errors on lifetime estimation assuming constant temperature measurement error $\left(N\left(\Theta_{\mathrm{TO}}, 5\right)\right)$ and process noise $(N(0,20))$.

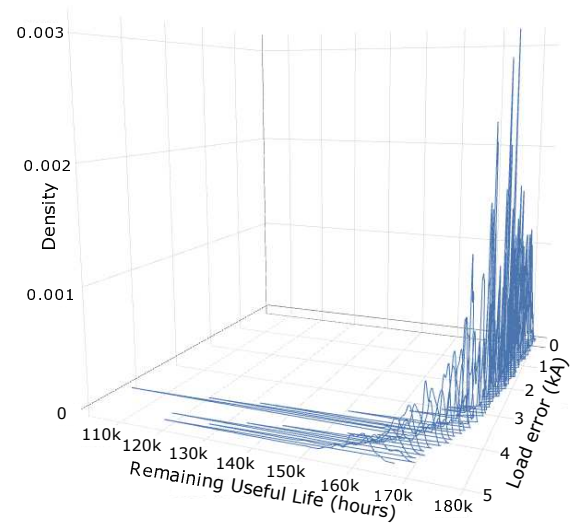

Fig. 10. Load error sensitivity analysis - 3D representation

It is apparent from Fig. 10 that different load measurement errors play a different role on the lifetime estimation. Fig. 11 shows the maximum likelihood and 95\% CI for the load error sensitivity analysis inferred from Fig. 10.

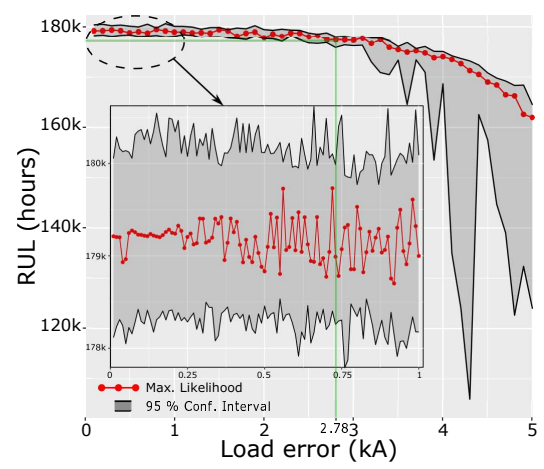

Fig. 11. Load error sensitivity analysis with $95 \% \mathrm{Cls}$

As the load measurement error magnitude increases in Fig. 11, the uncertainty bounds increase and the maximum likelihood value decreases. The zoomed view of the interval [0.01-1] kA shows that the error bounds are around 2000 hours and they are fairly constant in this zone. However, around the elbow point identified in (15) the maximum likelihood value starts decreasing rapidly and the $95 \%$ confidence intervals widen due to the increased effect of the error values. Owing to the stochastic nature of the PF algorithm, the $95 \%$ CIs vary according to the nature of the PDF (see PDFs in Fig. 10).

In order to evaluate the effect of temperature errors, the load measurement error $(N(i(t), 1))$ and process noise $(N(0,20))$ have been assumed constants. Fig. 12 shows the $95 \%$ CI for effect of error measurements for this situation.

In Fig. 12 one can see that for temperature measurement error values below $5^{\circ} \mathrm{C}$, the effect of temperature measurement errors on the RUL estimation is unstable. That is, the maximum likelihood value of the PDF of the RUL value fluctuates around $R U L_{0}=N(180000,500)$ minus the ageing after one year. Depending on the initial state which is randomly sampled from $R U L_{0}$, the final health state varies. There are some cases where the initial RUL is located around 180500 hours and therefore, after a year with a degradation lower than 500 hours, the final health state is above 180000 hours. 


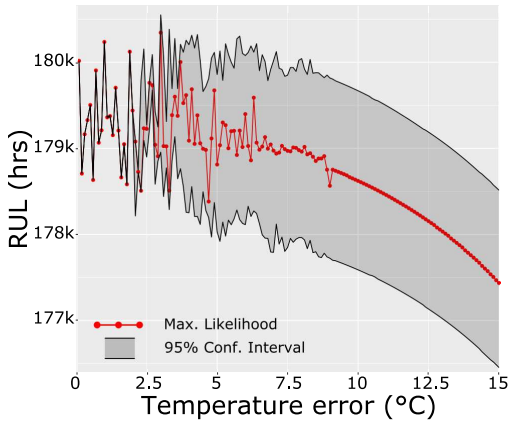

Fig. 12. Temperature error sensitivity analysis

On the other hand, above a temperature error of $5^{\circ} \mathrm{C}$ the effect of the temperature error becomes non-negligible and it directly affects the health state. Additionally, it is possible to see that for temperature measurement error values below $2{ }^{\circ} \mathrm{C}$ the CIs are very narrow, but as the error increases these bounds widen. This is because for low temperature errors the model is confident that the final health state is the maximum likelihood value because there is no temperature error. However, as the temperature error increases, the CIs widen and the final evaluation of the health state is more uncertain.

When the load error is zero and the temperature measurement error is $5^{\circ} \mathrm{C}$ (Fig. 11) the variation of the RUL estimation is caused by the term $\varphi_{\text {TO }}$ in (15). In contrast, when the temperature measurement error is zero in Fig 12, but the load error is kept at $1 \mathrm{kA}$, it can be seen that the variation due to the term $\varphi_{\mathrm{i}}$ in (15) is almost negligible, which confirms that the effect of temperature errors are more sensitive than load errors for low loading conditions.

\section{B. Prognostics}

In order to predict the future health state of the transformer the approach shown in Fig. 2 is adopted. First an appropriate predictive model is designed which is able to estimate HST given hypothetical load and temperature profiles. This estimation can then be directly connected with the PF framework to propagate uncertainties and estimate the lifetime. The adopted temperature error is $\varphi_{\mathrm{TO}}=5^{\circ} \mathrm{C}$ and load error $\varphi_{\mathrm{i}}=1 \mathrm{kA}$.

1) Feature processing \& selection: The length of the segment determines the validity of features and the final prediction error. According to the performed experiments, best results were obtained with a length of 5 days. With a segment length longer than this the features lost representativeness and the error increases (see Fig. 13) .

Subsequently, all the features (cf. Table I) along with the preprocessed variables have been processed through a recursive feature elimination (RFE) procedure and grid search [35]. This step selects the most representative features which minimize the prediction error. RFE was implemented for RF, XGB and SVR using the Caret $\mathrm{R}$ package and grid search was implemented for ANN and LSTM models. The error is quantified through 10 repeated 5 fold $\mathrm{CV}$ using the normalised root-mean-squared error $(\overline{R M S E})$ :

$$
\overline{R M S E}=\frac{R M S E}{\max \left\{R M S E_{\mathrm{i}}\right\}_{i=1}^{N}} ; R M S E=\sqrt{\frac{\sum_{i=1}^{N}\left(\hat{\Theta}_{\mathrm{TO}}-\Theta_{\mathrm{TO}}\right)^{2}}{N}}
$$

where $N$ is the number of predicted data samples.

Fig. 13 shows the feature selection results with best features for different segment sizes for XGB and RF models. Best results were obtained with the listed 12 features in Fig. 13.

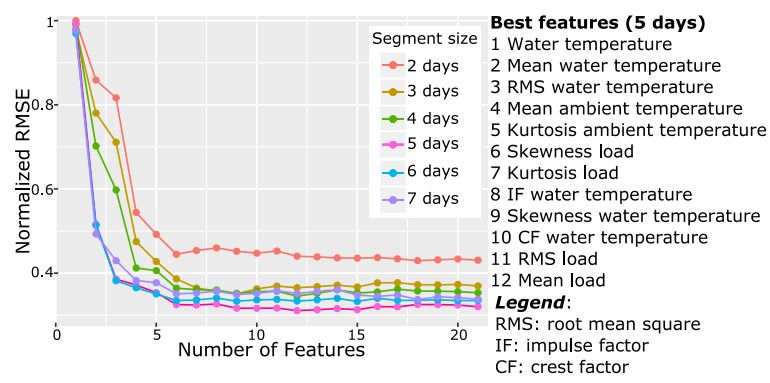

Fig. 13. Feature selection and segment size

Best results for SVR were obtained with three features (water temperature, mean ambient temperature, load), nine for ANN models (water temperature, mean ambient temperature, mean load, RMS water temperature, mean water temperature, skewness water temperature, RMS load, kurtosis ambient temperature, IF ambient temperature) and six for LSTM models (water temperature, mean ambient temperature, load, RMS load, mean load, mean water temperature) all with a segment size of 5 days. After the feature extraction step, all the forecasting models have been designed and trained according to the process outlined in Subsections III-B1-III-B5.

2) Thermal modelling: The first step is to learn a predictive model so as to predict the top-oil temperature. Figs. 5 and 14 show load, ambient, water and top-oil measurements hourly sampled for a period of 3 years and 10 months.

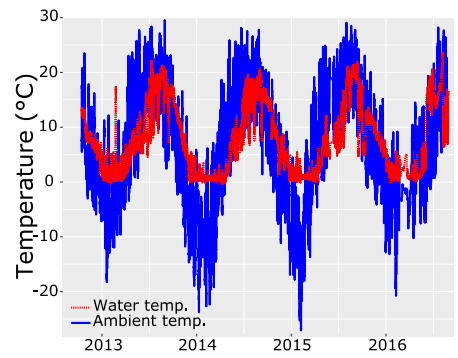

Fig. 14. Water and ambient temperature data (11/2012-09/2016)

It can be seen that the top-oil temperature profile is highly non-linear due to the specific operational constraints of NPPs. Namely, the plant is shut down for maintenance activities and this affects the load and top-oil temperature values. Additionally, depending on the harshness of the winter, load conditions and applied water temperature, the oil temperature can drop below zero degrees, e.g. winter 2016.

The learning process includes a 10 repeated 5 fold CV procedure to estimate parameters and generalize the prediction results. That is, the top-oil time series is divided into 5 equidistant folds (see Fig. 5). Then a number of ML models are trained (see Subsection III-B) for the first fold and tested with the second fold, subsequently the same models are trained with the first two folds and then tested with the third fold and the validation continues until the last step, where the models are trained with the first four folds and tested on the last fold. This process is repeated 10 times to deal with the stochastic 
behaviour of some models and generate repeatable results. For the error calculation the RMSE has been used. Note that there are different alternatives to validate the results such as the stratified double CV scheme [26].

In total, after preprocessing the data and removing invalid samples, there are 32440 samples so each fold has 6488 samples. Accordingly, at each fold the models predict up to 6488 hours ahead ( $~ 271$ days). Table III displays the mean RMSE and the standard deviation for various models for all the folds estimated through the 10 repeated 5 fold $\mathrm{CV}$ procedure.

TABLE III

RMSE OF ML MODELS FOR TOP-OIL TEMPERATURE FORECASTING

\begin{tabular}{|c|c|c|c|c|c|c|c|c|c|c|}
\hline \multirow{2}{*}{ Tech. } & \multicolumn{2}{|c|}{ Fold \#1 } & \multicolumn{2}{|c|}{ Fold \#2 } & \multicolumn{2}{|c|}{ Fold \#3 } & \multicolumn{2}{|c|}{ Fold \#4 } & \multicolumn{2}{|c|}{ Average } \\
\hline & $\mathrm{e}_{\text {train }}$ & $e_{\text {test }}$ & $\mathrm{e}_{\text {train }}$ & $\mathrm{e}_{\text {test }}$ & $e_{\text {train }}$ & $e_{\text {test }}$ & $e_{\text {train }}$ & $\mathrm{e}_{\text {test }}$ & $\mathrm{e}_{\text {train }}$ & $e_{\text {test }}$ \\
\hline \multirow{3}{*}{ XGB } & 0.37 & 4.13 & 1.2 & 5.07 & 2.08 & 6.63 & 3.04 & 9.1 & 1.67 & 6.23 \\
\hline & \pm & \pm & \pm & \pm & \pm & \pm & \pm & \pm & \pm & \pm \\
\hline & 0.28 & 0.37 & 0.35 & 0.35 & 0.13 & 0.67 & 0.24 & 0.15 & 1.14 & 2.17 \\
\hline \multirow{3}{*}{$\begin{array}{l}\text { LSTM } \\
(2 \mathrm{~L})\end{array}$} & 1.77 & 3.99 & 2.45 & 4.13 & 2.89 & 6.85 & \begin{tabular}{|l|}
4.63 \\
\end{tabular} & 9.97 & 2.94 & 6.24 \\
\hline & - & 土 & \pm & \pm & \pm & \pm & \pm & \pm & \pm & \pm \\
\hline & 0.05 & 0.42 & 0.12 & 0.3 & 0.15 & 0.25 & 0.28 & 0.39 & 1.05 & 2.43 \\
\hline \multirow{3}{*}{$\begin{array}{l}\text { LSTM } \\
(3 \mathrm{~L})\end{array}$} & 1.82 & 4.31 & 2.43 & 4.39 & 3.01 & 6.67 & 4.21 & 9.7 & 2.87 & 6.27 \\
\hline & \pm & \pm & \pm & \pm & \pm & \pm & \pm & \pm & \pm & \pm \\
\hline & 0.09 & 029 & 0.33 & 0.24 & 0.28 & 0.24 & 0.6 & 0.11 & 0.88 & 2.19 \\
\hline \multirow{3}{*}{$\begin{array}{l}\text { LSTM } \\
\text { (1L) }\end{array}$} & 2 & 4.3 & 2.52 & 4.4 & 3.29 & 6.9 & 4.34 & 9.6 & 3.05 & 6.3 \\
\hline & \pm & \pm & \pm & \pm & \pm & \pm & \pm & \pm & \pm & \pm \\
\hline & 0.17 & 0.71 & 0.24 & 0.49 & 0.52 & 0.26 & 0.67 & 0.52 & 1.01 & 2.5 \\
\hline \multirow{3}{*}{$\mathrm{RF}$} & 0.85 & 4.23 & 0.84 & 5.05 & 0.91 & 6.49 & 1.07 & 10 & 0.92 & 6.4 \\
\hline & \pm & \pm & \pm & \pm & \pm & \pm & \pm & \pm & \pm & \pm \\
\hline & 0.02 & 0.05 & 0.01 & 0.06 & 0.005 & 0.001 & 0.005 & 0.005 & 0.1 & 2.55 \\
\hline \multirow{3}{*}{ SVR } & & & & & & & & & 2.35 & 6.43 \\
\hline & 1.66 & 4.9 & 2.87 & 4.13 & 2.38 & 6.93 & 3.5 & 9.76 & \pm & \pm \\
\hline & & & & & & & & & 0.77 & 2.51 \\
\hline \multirow{3}{*}{ ANN } & 1.12 & 5.8 & 1.49 & 5.47 & 2.07 & 6.54 & 3.43 & \begin{tabular}{ll|}
11.07 \\
\end{tabular} & 2.03 & 7.22 \\
\hline & \pm & \pm & \pm & & \pm & \pm & \pm & \pm & \pm & \pm \\
\hline & 1.04 & 1.6 & 0.27 & 0.83 & 0.14 & 0.18 & 0.19 & 0.93 & 1.01 & 2.6 \\
\hline $\begin{array}{l}\text { IEEE } \\
\text { C57.91 }\end{array}$ & N/A & 5 & N/A & 5.6 & N/A & 8.6 & N/A & 12 & N/A & $\begin{array}{c}7.77 \\
\pm \\
3.2\end{array}$ \\
\hline
\end{tabular}

From the prediction results in Table III it can be seen that the XGB predicts best the top-oil temperature value. The mean performance of the LSTM is practically the same, but in the worst case scenario the maximum error is greater than the XGB, i.e. $e_{\text {test }_{\mathrm{XGB}}}=8.4<e_{\text {test }_{\mathrm{LSTM}}}=8.67$. Additionally, an important advantage of XGB over LSTM is that XGB models are easier and faster to train and test. Accordingly, the XGB model is used for lifetime modelling and RUL estimation. Fig. 15 shows the last fold prediction for XGB and LSTM, where ground truth denotes the measured top-oil temperature data.

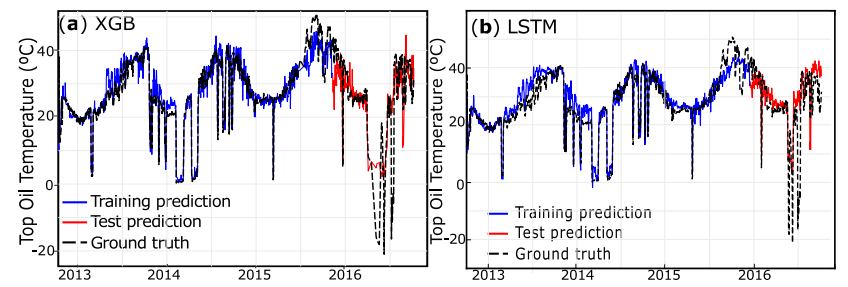

Fig. 15. Top-oil temperature forecasting results for the last fold (see top-oil temperature and folds in Fig. 5)

RF also shows a good performance, but the problem is that RF overfits the model as shown by the low training error. Also note that different trials of SVR models generate same results because of the fixed decision boundaries.

In contrast, the thermal model defined in the IEEE C57.91 standard has the poorest performance and highlights that the
IEEE analytic model may not perform accurately for every transformer operating in different contexts.

3) Lifetime modelling: The lifetime prediction model uses the most accurate thermal model within the framework in Fig. 2. Given hypothetical ambient temperature, load and water temperature variables, first the selected features are inferred (cf. Fig. 13), and then the XGB model predicts the topoil temperature. Subsequently, the IEEE model is used to estimate the hot-spot temperature from the predicted top-oil temperature, and finally, this is used to predict the paper RUL using the PF framework defined in Algorithm 1.

To test the approach with different hypothetical profiles, one year's worth top-oil and ambient temperature data have been taken from Fig. 14 as a representative reference for yearly temperature patterns. Then user-defined load profiles are used to predict the TOT under different loading conditions. Fig. 16 shows tested load and temperature patterns.
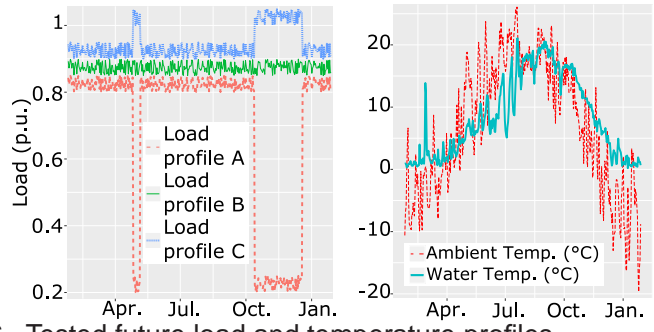

Fig. 16. Tested future load and temperature profiles

These patterns have been repeatedly applied to the PF framework in Algorithm 1 for two different prediction horizons of 5 and 10 years:

- Iine 1: initial state: $R U L_{0} \sim N(180000,500)$.

- line 4: infer selected features from load, ambient temperature and water temperature profiles in Fig. 16.

- Iine 5: using the designed XGB model, first forecast the TOT and then calculate the HST.

- line 6: draw random numbers corresponding to the assumed error variables.

- Iines 7-13: calculate the PDF of the RUL at time instant $t$.

This process is repeated for the selected prediction horizon (line 2) with a $\Delta t=1$ hour timestep, and finally the PDF of the RUL is obtained after processing the data up until the prediction horizon (line 14). Fig. 17 shows the RUL predictions after 5 and 10 years.
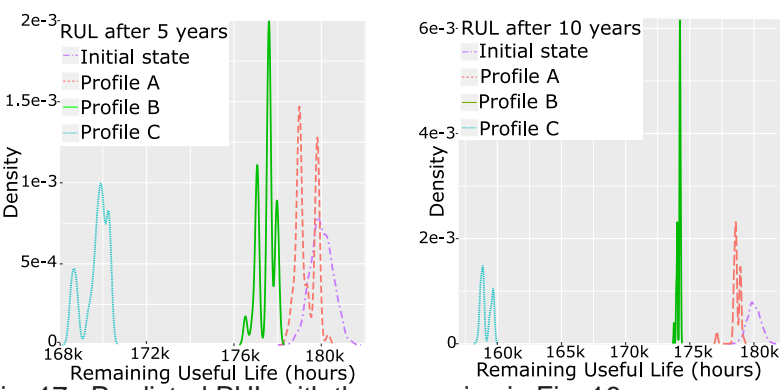

Fig. 17. Predicted RUL with the scenarios in Fig. 16

The initial state statistics in Fig. 17 are mean=179800, $95^{+}=180900,95^{-}=178900$ (all in hours). Table IV displays RUL statistics corresponding to different profiles. 
TABLE IV

RUL STATISTICS IN FIG. 17

\begin{tabular}{|c|ccc|ccc|ccc|}
\hline \multirow{2}{*}{ Time } & \multicolumn{3}{|c|}{ Profile A } & \multicolumn{3}{c|}{ Profile B } & \multicolumn{3}{c|}{ Profile C } \\
\cline { 2 - 9 } & $m$ & $95^{+}$ & $95^{-}$ & $m$ & $95^{+}$ & $95^{-}$ & $m$ & $95^{+}$ & $95^{-}$ \\
\hline $5 \mathrm{y}$ & $178.9 \mathrm{k}$ & $180.1 \mathrm{k}$ & $178.6 \mathrm{k}$ & $177.6 \mathrm{k}$ & $178.1 \mathrm{k}$ & $176.5 \mathrm{k}$ & $169.9 \mathrm{k}$ & $170.4 \mathrm{k}$ & $168.4 \mathrm{k}$ \\
\hline $10 \mathrm{y}$ & $178.5 \mathrm{k}$ & $179.1 \mathrm{k}$ & $177.1 \mathrm{k}$ & $174.2 \mathrm{k}$ & $174.3 \mathrm{k}$ & $173.7 \mathrm{k}$ & $158.9 \mathrm{k}$ & $159.8 \mathrm{k}$ & $158.4 \mathrm{k}$ \\
\hline
\end{tabular}

The predicted RUL values are consistent with the applied profiles. That is, $\mathrm{C}$ shows the most severe degradation followed by $\mathrm{B}$, and the application of A results in a higher RUL.

\section{CONCLUSIONS}

This paper has presented a novel transformer condition assessment approach integrating model-based experimental models, forecasting models and uncertainty modelling concepts in a Bayesian Particle Filtering framework.

Error propagation and sensitivity analysis are key activities for decision-making under uncertainty. The implemented sensitivity analysis evaluated the effect of load and temperature measurement errors on transformer lifetime and it showed that for low load measurement errors the effect of temperature errors are more critical. However, the load measurement error increases rapidly above an elbow value which has been formulated analytically.

It has been demonstrated that the integration of machine learning (ML) models with experimental models improves transformer lifetime estimations. Among the tested ML models for thermal modelling, the eXtreme Gradient Boosting (XGB) has shown the best prediction performance. Accordingly, the transformer RUL has been examined with different operational profiles using the XGB-based temperature prediction model, IEEE-based lifetime model and uncertainty information of collected measurements and stochastic processes. The predicted RUL values are consistent with the applied operational profiles and this demonstrates the validity of the proposed approach for adaptive lifetime predictions.

As NPPs age, the aging of transformers is becoming increasingly critical because they are crucial assets to export energy from the NPP. The proposed approach enables the modelling of these dynamic contexts accurately while accounting for uncertainties. Future work may focus on integrating other degradation accelerating factors in the proposed approach such as the moisture and other chemical factors.

\section{REFERENCES}

[1] W. H. Tang and Q. Wu, Condition monitoring and assessment of power transformers using computational intelligence. Springer, London, 2011.

[2] M. Liserre, G. Buticchi, M. Andresen, G. Carne, L. Costa, and Z. Zou, "The smart transformer: Impact on the electric grid and technology challenges," IEEE Ind. Electron. Mag., vol. 10, no. 2, pp. 46-58, 2016.

[3] H. Ma, T. K. Saha, C. Ekanayake, and D. Martin, "Smart transformer for smart grid - intelligent framework and techniques for power transformer asset management," IEEE Trans. Smart Grid, vol. 6, no. 2, pp. 10261034,2015

[4] J. Aizpurua, V. Catterson, B. Stewart, S. McArthur, B. Lambert, B. Ampofo, G. Pereira, and J. Cross, "Determining appropriate data analytics for transformer health monitoring," in NPIC-HMIT, pp. 1-11, 42017.

[5] M. Djamali and S. Tenbohlen, "Hundred years of experience in the dynamic thermal modelling of power transformers," IET Generation, Transmission Distribution, vol. 11, no. 11, pp. 2731-2739, 2017.

[6] A. Teymouri and B. Vahidi, "Co2/co concentration ratio: A complementary method for determining the degree of polymerization of power transformer paper insulation," IEEE Elect. Insul. Mag., vol. 33, no. 1, pp. 24-30, 2017.
[7] IEEE PES, "IEEE Guide for Loading Mineral-Oil-Immersed Transformers and Step-Voltage Regulators," IEEE Std. C57.91, 2011.

[8] R. Medina, A. Romero, E. Mombello, and G. Ratta, "Assessing degradation of power transformer solid insulation considering thermal stress and moisture variation," Electr. Pow. Syst. Res., vol. 151, pp. 1-11, 2017.

[9] Y. Cui, H. Ma, T. Saha, C. Ekanayake, and D. Martin, "Moisturedependent thermal modelling of power transformer," IEEE Trans. Pow. Del., vol. 31, no. 5, pp. 2140-2150, 2016.

[10] A. Seier, P. D. H. Hines, and J. Frolik, "Data-driven thermal modeling of residential service transformers," IEEE Trans. Smart Grid, vol. 6, no. 2 , pp. 1019-1025, 2015.

[11] E. Pournaras and J. Espejo-Uribe, "Self-repairable smart grids via online coordination of smart transformers," IEEE Trans. Ind. Infor., vol. 13, no. 4, pp. 1783-1793, 2017.

[12] M. Andresen, V. Raveendran, G. Buticchi, and M. Liserre, "Lifetimebased power routing in parallel converters for smart transformer application," IEEE Trans. Ind. Electron., vol. 65, no. 2, pp. 1675-1684, 2018.

[13] J. Velasquez, M. Sanz, and S. Galceran, "General asset management model in the context of an electric utility: application to power transformers," Electr. Pow. Syst. Res., vol. 81, no. 11, pp. 2015-2037, 2011.

[14] D. Villacci, G. Bontempi, A. Vaccaro, and M. Birattari, "The role of learning methods in the dynamic assessment of power components loading capability," IEEE Trans. Ind. Electron., vol. 52, no. 1, pp. 280290, Feb. 2005

[15] M. Hell and P. Costa and F. Gomide, "Participatory learning in power transformers thermal modeling," IEEE Trans. Pow. Del., vol. 23, no. 4, pp. 2058-2067, Oct. 2008.

[16] L. Souza, A. Lemos, W. Caminhas, and W. Boaventura, "Thermal modeling of power transformers using evolving fuzzy systems," Engineering Applications of Artificial Intelligence, vol. 25, no. 5, pp. 980 - 988, 2012.

[17] A. Bracale, G. Carpinelli, M. Pagano, and P. D. Falco, "A probabilistic approach for forecasting the allowable current of oil-immersed transformers," IEEE Trans. Pow. Del., vol. PP, no. 99, pp. 1-1, 2018.

[18] Q. Chen and D. M. Egan, "A bayesian method for transformer life estimation using perks' hazard function," IEEE Trans. Pow. Sys., vol. 21, no. 4, pp. 1954-1965, 2006.

[19] D. Zhou, Z. Wang, and C. Li, "Data requisites for transformer statistical lifetime modelling - part I: Aging-related failures," IEEE Trans. Pow. Del., vol. 28, no. 3, pp. 1750-1757, 2013.

[20] E. Chiodo, D. Lauria, F. Mottola, and C. Pisani, "Lifetime characterization via lognormal distribution of transformers in smart grids: Design optimization," Applied Energy, vol. 177, pp. 127 - 135, 2016.

[21] M. Humayun, M. Z. Degefa, A. Safdarian, and M. Lehtonen, "Utilization improvement of transformers using demand response," IEEE Trans. Pow. Del., vol. 30, no. 1, pp. 202-210, 2015.

[22] V. M. Catterson, J. Melone, and M. S. Garcia, "Prognostics of transformer paper insulation using statistical particle filtering of on-line data," IEEE Elect. Insul. Mag., vol. 32, no. 1, pp. 28-33, Jan. 2016.

[23] L. Jauregui-Rivera, X. Mao, and D. Tylavsky, "Improving reliability assessment of transformer thermal top-oil model parameters estimated from measured data," IEEE Trans. Pow. Del., vol. 24, no. 1, pp. 169-176, 2009.

[24] W. H. Tang, K. Spurgeon, Q. H. Wu, and Z. J. Richardson, "An evidential reasoning approach to transformer condition assessments," IEEE Trans. Pow. Del., vol. 19, no. 4, pp. 1696-1703, 2004.

[25] T. W. Rauber, F. Boldt, and F. M. V. ao, "Heterogeneous feature models and feature selection applied to bearing fault diagnosis," IEEE Trans. Ind. Electron., vol. 62, no. 1, pp. 637-646, Jan. 2015.

[26] R. Razavi-Far, M. Farajzadeh-Zanjani, and M. Saif, "An integrated classimbalanced learning scheme for diagnosing bearing defects in induction motors," IEEE Trans. Ind. Infor, vol. 13, no. 6, pp. 2758-2769, 2017.

[27] L. Breiman, "Random forests," Machine Learning, vol. 45, no. 1, pp. 5-32, Oct. 2001.

[28] M. R. G. Meireles, P. E. M. Almeida, and M. G. Simoes, "A comprehensive review for industrial applicability of artificial neural networks," IEEE Trans. Ind. Electron., vol. 50, no. 3, pp. 585-601, Jun. 2003.

[29] A. J. Smola and B. Schölkopf, "A tutorial on support vector regression," Statistics and Computing, vol. 14, no. 3, pp. 199-222, Aug. 2004.

[30] T. Chen and C. Guestrin, "Xgboost: A scalable tree boosting system," in Proc. of ACM Knowledge Discov. \& Data Mining, pp. 785-794, 2016.

[31] S. Hochreiter and J. Schmidhuber, "Long short-term memory," Neural Computation, vol. 9, no. 8, pp. 1735-1780, 1997.

[32] J. Friedman, T. Hastie, and R. Tibshirani, "Additive logistic regression: a statistical view of boosting," Ann. Statist., vol. 28, no. 2, pp. 337-407, 2000. 
[33] M. S. Arulampalam, S. Maskell, N. Gordon, and T. Clapp, "A tutorial on particle filters for online nonlinear/non-gaussian bayesian tracking," IEEE Trans. Signal Process., vol. 50, no. 2, pp. 174-188, Feb. 2002.

[34] B. Saha and K. Goebel, "Model adaptation for prognostics in a particle filtering framework," Int. J. of Prognostics and Health Management, vol. 2, no. 6, 2011.

[35] M. Kuhn and K. Johnson, An Introduction to Feature Selection, pp. 487-519. New York, NY: Springer New York, 2013.

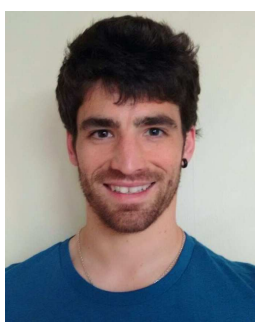

Jose Ignacio Aizpurua (M'17) is a Research Associate within the Institute for Energy and Environment at the University of Strathclyde, Scotland, UK. He received his Eng., M.Sc., and Ph.D. degrees from Mondragon University (Spain) in 2010, 2012, and 2015 respectively. He was a visiting researcher in the Dependable Systems Research group at the University of Hull (UK) in 2014. His research interests include prognostics and health management, reliability, availability, maintenance and safety (RAMS) analysis and systems engineering for power engineering applications.

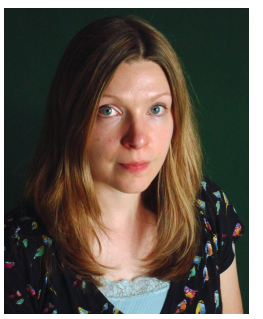

Victoria M. Catterson (M'06-SM'12) is a Senior Lecturer within the Institute for Energy and Environment at the University of Strathclyde, Scotland, UK. She received her B.Eng. (Hons) and $\mathrm{Ph}$.D. degrees from the University of Strathclyde in 2003 and 2007 respectively. Her research interests include condition monitoring, diagnostics, and prognostics for power engineering applications

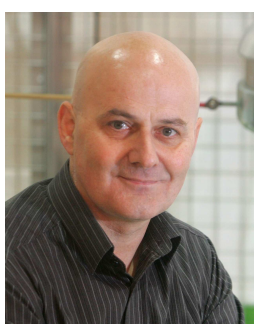

Brian G. Stewart (M'08) is is Professor within the Institute of Energy and Environment at the University of Strathclyde, Glasgow, Scotland. He graduated with a BSc (Hons) and PhD from the University of Glasgow in 1981 and 1985 respectively. He also graduated with a BD (Hons) in 1994 from the University of Aberdeen, Scotland. His research interests are focused on high voltage engineering, electrical condition monitoring, insulation diagnostics and communication systems. He is currently an AdCom Member within the IEEE Dielectrics and Electrical Insulation Society.

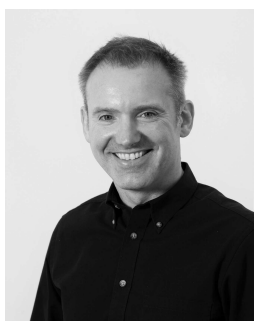

Stephen D. J. McArthur (M'93-SM'07-F'15) received the B.Eng. (Hons.) and Ph.D. degrees from the University of Strathclyde, Glasgow, U.K., in 1992 and 1996, respectively. He is a Professor and co-Director of the Institute for Energy and Environment at the University of Strathclyde. His research interests include intelligent system applications in power engineering, covering condition monitoring, diagnostics and prognostics, active network management and wider smart grid applications.

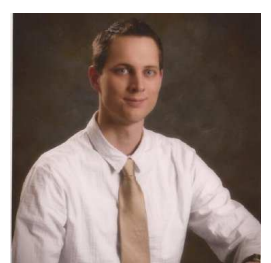

Brandon Lambert is a Design Engineering Manager within Bruce Power. He received his B.Eng. degree from Lakehead University, Thunder Bay, Canada in 2012 and his P.Eng. from the Professional Engineers of Ontario in 2015. His design interests include large power transformers, high voltage transmission systems, as well as dielectric and insulating materials.

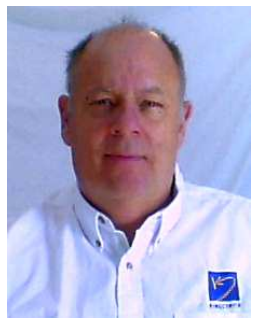

James Cross (M '79) is currently Director of Transformer Services at Kinectrics, In. in Toronto, Canada. After graduating from the University of Manitoba with a B.Sc. in Electrical Engineering, he worked for 18 years at Carte International, a transformer manufacturer in Winnipeg, Canada as Vice-President, Technology. He then worked as a Project Engineer at Pauwels Canada, a manufacturer of large power transformers up to $500 \mathrm{kV}$ class. Most recently, he worked for 18 years at Weidmann Electrical Technology in St. Johnsbury, Vermont serving as Manager of R\&D/Innovation and Manager of Technical Services. He has coauthored several papers in the area of electrical insulating materials and testing, and transformer diagnostics. He is a former Chairperson of the IEEE Winnipeg Section. 